University of Wollongong

Research Online

Faculty of Informatics - Papers (Archive)

Faculty of Engineering and Information

Sciences

$1-8-2006$

\title{
Ultra-wideband technology for video surveillance sensor networks
}

Xiaojing Huang

University of Wollongong, huang@uow.edu.au

Eryk Dutkiewicz

University of Wollongong, eryk@uow.edu.au

Ricardo Gandia

University of Wollongong

Darryn Lowe

University of Wollongong, darrynl@uow.edu.au

Follow this and additional works at: https://ro.uow.edu.au/infopapers

Part of the Physical Sciences and Mathematics Commons

\section{Recommended Citation}

Huang, Xiaojing; Dutkiewicz, Eryk; Gandia, Ricardo; and Lowe, Darryn: Ultra-wideband technology for video surveillance sensor networks 2006.

https://ro.uow.edu.au/infopapers/475

Research Online is the open access institutional repository for the University of Wollongong. For further information contact the UOW Library: research-pubs@uow.edu.au 


\title{
Ultra-wideband technology for video surveillance sensor networks
}

\author{
Abstract \\ Wireless sensor networks have received a lot of attention in recent years both in the research community \\ and in standards organizations. The emphasis of most sensor network research activities has been on \\ the development of low cost and low power technologies for low bit rate applications. In this paper, we \\ describe our work on ultra-wideband (UWB) sensor network technology targeted for video surveillance \\ applications that require high immunity to noise, interference and jamming, high bit rate, and quality of \\ service support. Such sensor networks are expected to find primary applications in the public safety and \\ military fields. Our emphasis in this paper is on the design of the physical layer that can support such \\ requirements and provide high end-to-end throughput when multi-hopping is used.

\section{Disciplines} \\ Physical Sciences and Mathematics

\section{Publication Details} \\ This paper was originally published as: Huang, X, Dutkiewicz, E, Gandia, R \& Lowe, D, Ultra-wideband \\ technology for video surveillance sensor networks, IEEE International Conference on Industrial \\ Informatics 2006, Singapore, August 2006, 1012-1017. Copyright IEEE 2006.
}




\title{
Ultra-Wideband Technology for Video Surveillance Sensor Networks
}

\author{
Xiaojing Huang, Eryk Dutkiewicz, Ricardo Gandia, Darryn Lowe \\ Wireless Technologies Laboratory \\ University of Wollongong \\ Wollongong NSW 2522 \\ AUSTRALIA \\ huang@uow.edu.au
}

\begin{abstract}
Wireless sensor networks have received a lot of attention in recent years both in the research community and in standards organizations. The emphasis of most sensor network research activities has been on the development of low cost and low power technologies for low bit rate applications. In this paper, we describe our work on ultra-wideband (UWB) sensor network technology targeted for video surveillance applications that require high immunity to noise, interference and jamming, high bit rate, and quality of service support. Such sensor networks are expected to find primary applications in the public safety and military fields. Our emphasis in this paper is on the design of the physical layer that can support such requirements and provide high end-to-end throughput when multi-hopping is used.
\end{abstract}

\section{INTRODUCTION}

$\mathrm{W}$ IRELESS sensor network research has been progressing at a fast pace over recent years with the aim of developing technologies that will meet low cost, low power and high reliability requirements. Maturity of such research can be judged by the success of relevant standards organizations. In recent years the IEEE 802.15 Working Group has been considering new physical layers based on ultra-wideband (UWB) to support both low bit rate and high bit rate applications that include sensor networks. However, these efforts have had mixed success. An UWB physical layer sensor network standard is well under development in the IEEE 802.15.4 Task Group, however, attempts to standardize a high bit rate UWB physical layer in the IEEE 802.15.3a Task Group have been abandoned. In this paper, we describe our work on development of a physical layer (PHY) design that targets middle range applications that require high bit rates and quality of service support. Section II gives an overview of UWB technology and describes recent efforts in the IEEE standards to develop UWB physical layers. Section III describes the requirements for a new physical layer that can support medium range, high bit rate applications targeted for public safety and military applications. Section IV describes the design of the physical layer and presents preliminary performance results. Section $\mathrm{V}$ presents our conclusions.

\section{UWB SENSOR NETWORKS TECHNOLOGY}

\section{A. UWB Technology}

In its traditional spread spectrum definition, UWB is a unique radio technology different from its conventional narrowband counterpart by transmitting electromagnetic signals with relatively large proportion of frequency spectrum. The UWB signal demonstrates some unique properties that make this technology useful in a large range of applications in many different ways. For example, its resistance to interference and interception has attracted a lot of attention from the military for use in secure short-tomedium distance $(<1 \mathrm{~km})$ communication applications. This is exemplified by the "NETEX" R\&D program launched by DARPA [1]. This program has also given motivation to our research focus. The ability of an UWB signal to penetrate through objects may allow the mining industry to use it for ground-penetrating detection and the emergency services for through-wall motion sensors. Moreover, the high time resolution properties of an UWB radio could be used to construct precision location systems with sub-centimeter resolutions. As a result, UWB technology can find wide commercial opportunities in wireless local area network (WLAN) and wireless personal area network (WPAN), mobile communications, wireless audio/video, automotive, and many more.

The early work on UWB communications was performed under classified US Department of Defense programs. In response to the growing practicality of UWB-based communications, the Federal Communications Commission (FCC) in the USA released a Notice of Inquiry in September, 1998 to investigate the possibility of permitting the operation of unlicensed UWB devices under its Part 15 rules. In May, 2000, the FCC released a Notice of Proposed Rulemaking that began the process of identifying potential rule changes necessary for the deployment of UWB technology. After the FCC eventually adopted its First Report and Order in February 2002 [2], that permitted the marketing and operation of UWB technology, UWB communications became legal as long as the mean transmit power does not exceed $-41.3 \mathrm{dBm} / \mathrm{MHz}$ in the frequency range $3.1 \mathrm{GHz}$ to 10.6 GHz. More recently the FCC issued a further ruling with regards to transmission power measurement compliance. This ruling appears to further relax the use of UWB in the USA. 
Typical UWB systems include the time-hopping UWB systems [3], direct sequence (DS) UWB systems [4] and the multi-band orthogonal frequency division multiplexing (OFDM) UWB systems [5]. To mitigate intersymbol interference (ISI) in multipath channels, the conventional DS UWB systems have to resort to complicated linear equalization or decision feedback equalization techniques which are too costly for low power low cost applications. When operating in severe multipath environments, the other UWB systems demonstrate a major disadvantage that they either do not provide adequate ISI mitigation or require complicated equalization techniques to reduce ISI. The UWB PHY presented in this paper overcomes these disadvantages.

\section{B. Standardization Efforts}

The main standards driving force behind the UWB-based physical layer for wireless communications is the IEEE 802.15.3 standards working group. With its main focus in the USA, this standards organization is responding to the market opportunities that have been presented by the FCC approval in the USA.

The 802.15.3a task group was established in 2003. Following the IEEE 802 standards process of proposal downselection, two contenders for the high-rate standards were vying for the standard. These were the DS-UWB and MBOFDM technologies. Fig. 1 shows the timeline for the standardization process within the 802.15.3a task group. The standardization process was abandoned in January 2006.

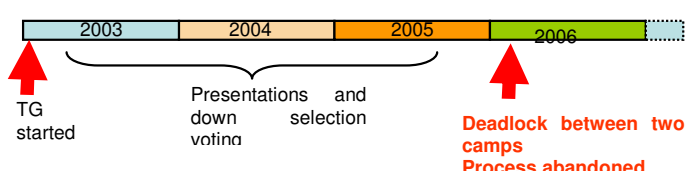

Fig. 1. Timeline of the IEEE 802.15.3a Task Group.

The DS-UWB proposal [4] is based on the spread spectrum principle. It uses two operating bands: Low Band between 3.1 and $4.9 \mathrm{GHz}$ and High Band between $6.2 \mathrm{GHz}$ and $9.7 \mathrm{GHz}$. The High Band is optional and currently not implemented. Each band can support up to 6 piconets. Each piconet is defined as a group of communicating devices within the range of the controlling device (called the Piconet Controller). Data rates supported in the Low Band range from $28 \mathrm{Mbps}$ to $1 \mathrm{Gbps}$. The High Band can reach up to 2 Gbps.

The MB-OFDM proposal [5] uses OFDM techniques. It can support between 4 to 16 simultaneous piconets and it can achieve data rates from $53 \mathrm{Mbps}$ to $480 \mathrm{Mbps}$. The available UWB spectrum is divided into 14 bands, each $528 \mathrm{MHz}$ wide, grouped into 5 band groups. Band Group 1, consisting of the first three bands is mandatory with the remaining bands reserved for the future. Devices using Band Group 1 can support up to 4 simultaneous piconets. Each piconet is assigned a different logical channel which corresponds to a unique time frequency code. The time frequency code determines the hopping sequence of bands used by the piconet. Potential interference between the four overlapping piconets is reduced for data rates up to $200 \mathrm{Mbps}$ through the use of time-domain spreading by transmitting the same information over two OFDM symbols. The amount of dollars invested by both camps to produce silicon and the expectations of a potentially multi-billion dollars industry enabled by this technology may mean that the standards process will fail and the battle for supremacy will be fought in the market place.

The 802.15.4a task group was established in 2004 . Following the IEEE 802 standards process of proposal downselection, a merged spread spectrum based proposal is currently being drafted into the standard. The complete standard is expected in 2007. This is in stark contrast to the abandoned process for the high-rate UWB standard in the IEEE 802.15.3a task group. Fig. 2 shows the timeline for the standardization process within the 802.15.4a task group.

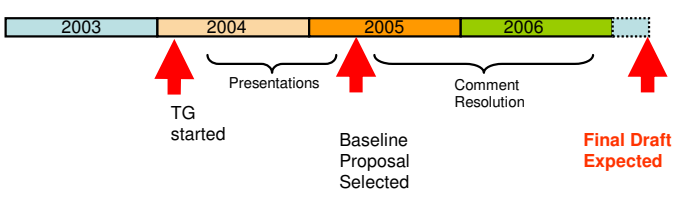

Fig. 2. Timeline of the IEEE 802.15.4a Task Group.

The requirements for the low-rate UWB PHY include precision ranging within tens of centimeters, communication distance of around $30 \mathrm{~m}$, support for bit rates of more than $1 \mathrm{kbps}$ on individual links and more than $1 \mathrm{Mbps}$ on aggregate links [6]. The proposed IEEE 802.15.4a standard will allow two communication modes: a UWB signaling mode in the UWB band (based on the DS-UWB technique similar to that proposed for the high-rate standard in the IEEE 802.15.3a task group) and a chirp signaling mode in the ISM band [7]. The chirp signaling mode in the ISM band will enable operation in markets where regulatory approvals are still under consideration for UWB. Precision ranging can only be supported with the UWB signaling mode, whereas both the UWB signaling and chirp signaling modes can be used for communication. Chirp signaling can also be used as an option with the UWB signaling mode to improve simultaneous overlapping piconet performance. The 802.15.4a standard is currently in a draft form and will be refined throughout 2006. Its release is expected in early 2007.

The two industry alliances behind the DS-UWB and MBOFDM technologies in the IEEE 802.15.3a task group are the UWB Forum and the WiMedia Alliance, respectively. Both the UWB Forum and WiMedia Alliance actively promote their respective flavors of UWB technology at major technology exhibitions and shows around the world and they both lobby the regulatory agencies around the world to permit the use of UWB technology without which their markets will not eventuate. The industry alliance promoting 
the low-rate personal area networks standards of IEEE 802.15.4 is the ZigBee Alliance. Given that the IEEE 802.15.4a task group has agreed on a combined proposal for drafting into a standard, it is expected that the ZigBee Alliance will also support this new standard.

The application scenarios targeted by the high bit rate and low bit rate UWB technologies described above offer an opportunity for development of a new mid-range high bit rate UWB physical layer for public safety and military applications. Such applications have also been targeted by DARPA's NETEX program [1].

\section{ULTRA-WIDEBAND PHY DESIGN}

\section{A. Ultra-Wideband PHY Requirements}

An UWB physical layer targeted for robust communications in public safety and military environments will need to provide an effective means to combat multipath interference and have the ability to coexist with legacy systems and intentional jammers. Support is also required for high bit rate real-time video and audio applications over long point-to-point distances, greatly exceeding the range available in current UWB systems. The PHY also has to be capable of overcoming severe throughput degradation present in current multi-hop UWB networks. The objective of our work has been to develop techniques that enable a UWB radio supporting a 10 Mbps data rate with binary modulation and up to $40 \mathrm{Mbps}$ data rate with multi-level modulation over a distance of 100 meters. The transmitted signal bandwidth is $1 \mathrm{GHz}$. The carrier frequency is $6 \mathrm{GHz}$.

\section{B. CCDM and MCIDS Baseband Schemes}

Fig. 3 shows a block diagram of an UWB transceiver. The key baseband techniques employed in the transceiver are Complementary Code Division Multiplexing (CCDM) [8] and Multi-Code Interleaved Direct Sequence (MCIDS) spreading [9]. CCDM makes the signal amplitude Gaussian distributed and enables scalable data rates. It also acts as a block coding step that makes conventional channel coding unnecessary and thereby reduces the complexity of decoding. MCIDS is a combination of multicode direct sequence spreading with block interleaving. After the input data symbols are divided into data blocks, each symbol in a block is spread by a specific spreading sequence. After all symbols in the block are spread by a set of orthogonal spreading sequences respectively, the spread signals are then passed through a block interleaver to produce the final MCIDS spread signal. A cyclic prefix may also be added at the beginning of the interleaved spread signal. The length of the cyclic prefix is required to be longer than the maximum channel delay. The chip time is set to $6.25 \mathrm{nS}$.

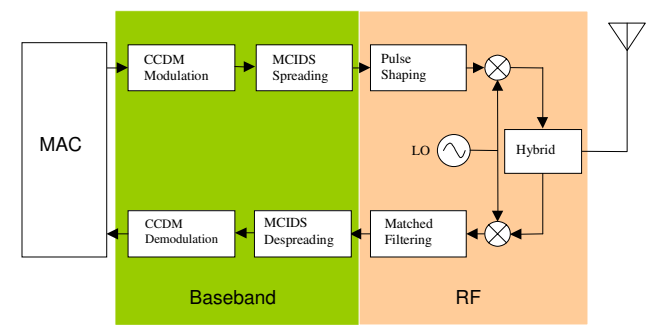

Fig. 3. Block Diagram of UWB Transceiver.

The received MCIDS spread signal can be despread by a circular despreading and combining technique. The ISI caused by multipath will be cancelled due to the orthogonality of the sequence set used in MCIDS spreading. The signal energy in each path can be collected by a RAKE receiver, realizing the highest degree path diversity reception. The MCIDS spreading offers ISI-free data transmission over severe multipath channels that can have a channel delay spread much longer than the symbol duration. This is a fundamental advantage over conventional direct sequence spreading, in which the channel delay spread must be only a fraction of the data symbol duration. Other advantages of the MCIDS spreading include a flatter signal spectrum and the ability to provide adaptive processing gains combined with CCDM.

The MCIDS exploits the same philosophy that OFDM uses to mitigate multipath, i.e., the data symbols are firstly grouped block by block and then mapped onto a set of orthogonal sequences with extended symbol duration. Thus, the ISI is cancelled due to the orthogonality of the sequence set. Innovatively, MCIDS uses a binary spreading sequence set which occupies a wide bandwidth and produces a flat signal spectrum, whereas in conventional OFDM the orthogonal sequences are complex exponentials which represent narrowband subcarriers in the frequency domain. To further spread the signal to wider bandwidth, the OFDM based UWB system has to use additional frequency hopping which adds to system complexity and causes transmit power compliance issues. Furthermore, the block interleaving used in MCIDS does not degrade the peak-to-average power ratio (PAPR) of the input signal provided by CCDM, whereas in OFDM the modulated subcarriers are simply summed up, which leads to a high PAPR.

\section{Concurrent Tx/Rx Capability}

Since the impulse signalling is used in the MCIDS spread spectrum scheme, the transmitted signal can be easily synchronized with the received signal using time-domain multiplexing by a transceiver node in an UWB network to form a pipelined packet transmission. Fig. 4 shows the signals that are present at the RF front-end of the first packet destination, i.e., Node B. There are four different types of signals at the receive path of Node B. One is the signal leakage from Node B's transmit path due to the non-ideal 
isolation of the hybrid. The other three are received from its antenna and can be classified as the signal transmitted from Node A (including multipath reflections), reflections from the Node B's own transmitted signal, and the signal transmitted from Node $\mathrm{C}$ (including multipath reflections). Since the only desired signal is the one from Node A, Node B must be able to reject the other signals as they are only sources of interference. We refer to the signal leakage and the reflections coming from Node B's transmission as selfinterference caused by the local transmitter, and the signal and reflections coming from Node C's transmission as interference caused by a remote transmitter. Thus, the receiver must be capable of eliminating the interference generated by both local and remote transmitters.

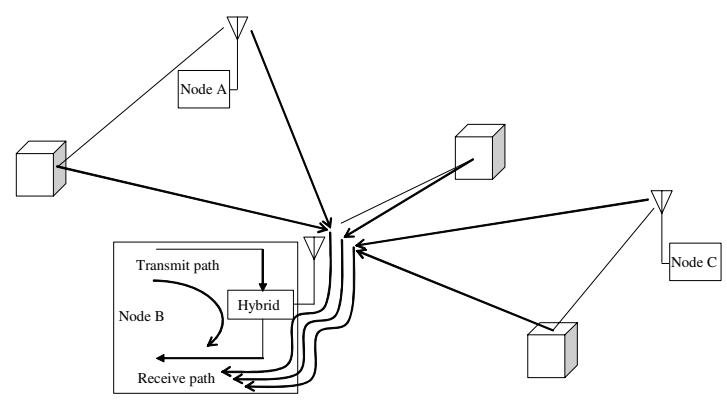

Fig. 4. Signals Present at RF Front End of Node B.

For the local transmitter interference cancellation, we use block data transmission with code-division outer codes. In this method, the data to be transmitted at a transceiver is grouped block by block. The block size is chosen so that the length of the transmitted signal waveform for one data block is significantly longer than the maximum channel delay spread. An outer code is then applied to the transmitted data blocks. These outer codes are managed so that the signal that a given transceiver transmits is orthogonal to the one that it is attempting to receive. For example, the simplest orthogonal outer code set is composed of the codes $\{+1,+1\}$ and $\{+1$, $-1\}$, where the code $\{+1,+1\}$ is applied to the transmitted signal blocks from a remote transmitter and the code $\{+1,-1\}$ is applied to the transmitted signal blocks from the local transmitter. After the transceiver removes the locally generated interference through despreading the code $\{+1$, $+1\}$, only the original remote signal remains. These outer codes are applied to the MCIDS blocks.

By exploiting the capability of the MCIDS spread spectrum technique to distinguish between signals arriving at different time delays, the remote transmitter interference can be also easily removed. In this method, the data to be transmitted is grouped block by block and then spread by the MCIDS spreading technique. The signal transmitted from Node $\mathrm{C}$ only needs to be delayed a certain amount of time relative to the signal transmitted from Node A to allow Node $\mathrm{B}$ to receive the signal from Node A only, and discard the signal from Node C. The reason why this can be done is because rather than attempting to resolve the delayed signal, as is the case when resolving multipath, the delayed signals in this case are simply discarded. The time delay introduced by Node $\mathrm{C}$ is one half of the maximum channel delay which the MCIDS scheme is designed to cope with.

\section{PHY Frame Format}

As shown in Fig. 5, the PHY frame contains three fields: preamble with start frame delimiter (SFD), header (including PHY header and MAC header) with header check sequence (HCS), and frame payload with frame check sequence (FCS). Stuff bits (SB) may be added following FSC in order to group payload and FCS bits into CCDM and MCIDS blocks.

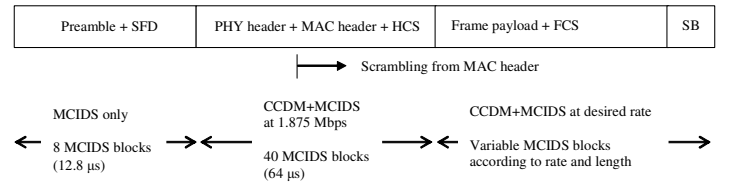

Fig. 5. PHY Frame Format.

The preamble with SFD field consists of 8 MCIDS blocks and lasts $12.8 \mu \mathrm{s}$. Only MCIDS spreading is applied in this field (i.e., no CCDM modulation). The header field consists of 40 MCIDS blocks under normal operation. The frame payload with FCS field contains variable MCIDS blocks according to the length of payload and the date rate defined in the PHY header. CCDM modulation starts from the PHY header. Data scrambling starts from the MAC header. There are three preamble options. Preamble option 1 is used for normal operation mode without concurrent Tx/Rx. Preamble option 2 is used for concurrent $\mathrm{Tx} / \mathrm{Rx}$ operation mode with $\{+1,+1\}$ outer code. Preamble option 3 is used for concurrent $\mathrm{Tx} / \mathrm{Rx}$ operation mode with $\{+1,-1\}$ outer code. There are also three SFD options in conjunction with the above defined preamble options.

The PHY header consists of three octets that specify the seed identifier for the data scrambler, the data rate of the frame payload, and the number of octets in the frame payload (which does not include FSC or SB). The number of MCIDS blocks produced by the frame payload (with FSC and SB) can be determined by the data rate and payload length information.

The nominal symbol rate before MCIDS spreading is 10 Msps. However, since 4 out of the 16 total symbols in a MCIDS block are used for pilots, the nominal data rate after MCIDS spreading is reduced to 7.5 Mbps. The PHY protocol defines six data rates: $1.875 \mathrm{Mbps}, 3.75 \mathrm{Mbps}$, 7.5 Mbps, 15 Mbps, $30 \mathrm{Mbps}$, and $45 \mathrm{Mbps}$. Each data rate is achieved by choosing a unique symbol mapping and symbol rate combination used in CCDM. The MAC header consists of 10 octets, which is the same as that defined in IEEE 803.15.3. The header field is transmitted using the lowest date rate of 1.875 Mbps.

The CCDM modulation uses a set of 8 orthogonal complementary code spreading sequences of length 64 . This 
set of orthogonal complementary code spreading sequences is used to modulate 8 data symbols. The size of one CCDM block is 96 . Before CCDM modulation, binary serial input data bits need to be divided into groups of $1,2,4$, or 6 bits and converted into data symbols (complex numbers representing BPSK, QPSK, 16-QAM, or 64-QAM constellation points) according to the data rate used.

MCIDS spreading uses the Walsh-Hadamard sequence set of length 16. One MCIDS block will spread 12 CCDM signal samples and 4 pilot bits. The pilot bits are defined as $\{+1$, $-1,+1,-1\}$. The pilot bits are located in position $0,4,8$, and 12. 8 MCIDS blocks are required to spread one CCDM block.

The PHY has two operation modes. One is the normal operation mode and the other is the concurrent $\mathrm{Tx} / \mathrm{Rx}$ operation mode. Under normal operation mode, preamble option 1 is used. No outer code is used for the MCIDS blocks in the header and payload fields of the PHY frame. Under concurrent $\mathrm{Tx} / \mathrm{Rx}$ operation mode, preamble option 2 is used if outer code $\{+1,+1\}$ is used or option 3 is used if outer code $\{+1,-1\}$ is used. When the transceiver starts to transmit while receiving a packet, the transmitter clock is delayed by half chip time (3.125 ns). The MCIDS block must be delayed by $1 / 4 \mathrm{~L}$ chip time, where $\mathrm{L}=16$ denoting the allowed maximum channel delay (in chip time). More detailed description of the PHY can be found in [10].

\section{E. Transceiver Algorithms}

In the transmitter, the CCDM modulation is used to map the information bits into symbols according to different data rates and spread these symbols over one CCDM block. By defining three different CCDM symbol rates and four different mapping constellations, six data rates can be supported. The MCIDS further spreads the CCDM modulated signal samples with a fixed spreading ratio in order to cope with multipath channels. If the transmitter is working under the concurrent $\mathrm{Tx} / \mathrm{Rx}$ mode, an outer code of $\{+1,+1\}$ or $\{+1,-1\}$ will be applied to the output MCIDS spread signal.

The function of the receiver is to search the SFD, parse the PHY header and detect the PSDU. Before the receiver can process the received signal digitally, it is first passed through a match-filter and sampled at 4 samples per chip. Since the channel is unknown before a frame of the signal arrives, the receiver should perform initial channel estimation and synchronization using the preamble before SFD search. Once the receiver detects a coming frame, a sequence, constructed by spreading the 16-bit synchronization sequence using MCIDS, is used to correlate the incoming signal samples. Due to the lack of MCIDS block timing at the start point, we need to perform the correlation for a number of offsets which is equal to the length of one MCIDS block plus the length of the maximum channel delay. The correlation output represents the multipath profile of the channel.

Once the multipath profile is obtained, we then truncate it to the predefined maximum channel length. After that, the synchronization goes to the tracking phase. After the receiver gets synchronized with the incoming signal frame, the MCIDS blocks in the frame are despread to retrieve the signal symbols. The MCIDS dispreading can be done using the circular despreading and combining technique described in [9]. The CCDM demodulation is the reverse operation of the CCDM modulation, which includes two major operations: despread the CCDM block to retrieve the symbols and de-map the symbols into binary data bits.

\section{PERFORMANCE EVALUATION}

Fig. 6 shows the block diagram of the system model used for the simulation. A BER analyzer is used to calculate the BER after the receiver detects the transmitted frames.

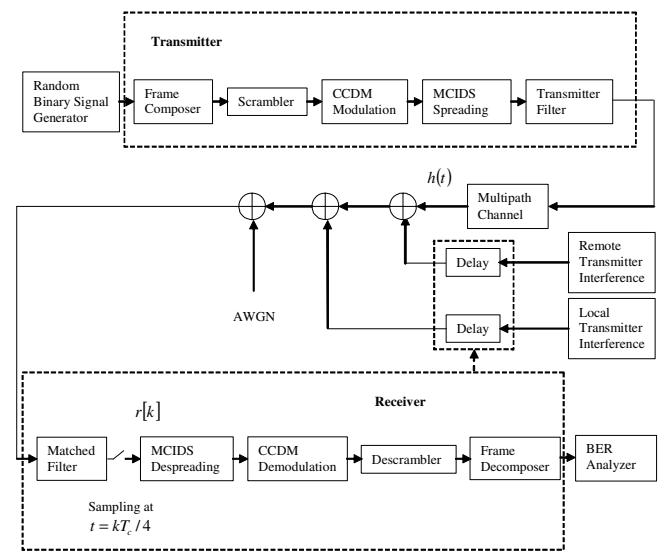

Fig. 6. System Simulation Model.

The algorithms described in previous section have been tested under different data rates and channel environments. Some simulation parameters are listed in Table 1.

The performance of the UWB receiver algorithms in Gaussian channel is shown in Fig. 7. The results prove that these algorithms are feasible in practice and the simulation platform is working. Compared with theoretical performance in Gaussian channel, there is a slight degradation for each data rate. This is because the synchronization is not perfect under noisy conditions and the channel estimation is also not perfect. In addition, there is a slight degradation for lower rate BPSK, as compared with QPSK. This indicates that the CCDM does not produce $100 \%$ coding gain without perfect channel estimation. 
TABLE I

SIMULATION PARAMETERS

\begin{tabular}{ll}
\hline \hline \multicolumn{1}{c}{ Parameter } & \multicolumn{1}{c}{ Parameter Value } \\
\hline Transmitter frame length & 1024 octets \\
Transmitter data rate & Varying according to modulation \\
Transmitter chip rate & $6.25 \mathrm{nS}$ \\
$\begin{array}{l}\text { Transmitter filter } \\
\text { sampling rate }\end{array}$ & 16 samples/chip \\
$\begin{array}{l}\text { Receiver sampling rate } \\
\text { (after matched filter) }\end{array}$ & 4 samples/chip \\
\end{tabular}

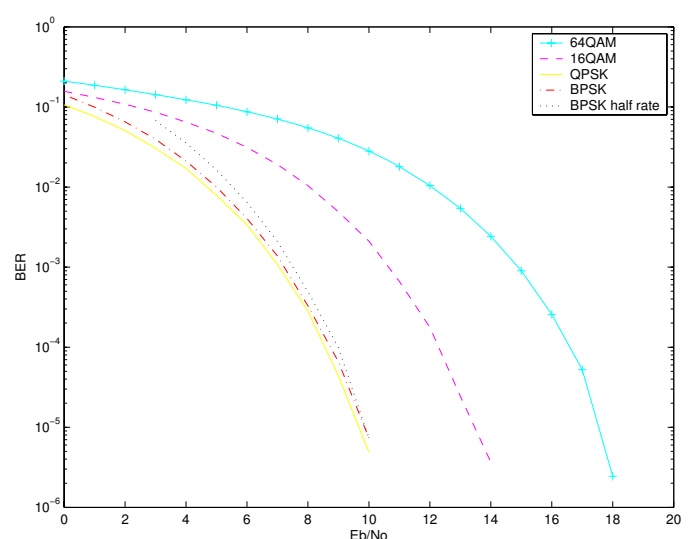

Fig. 7. Performance under Normal Operation in Gaussian Channel.

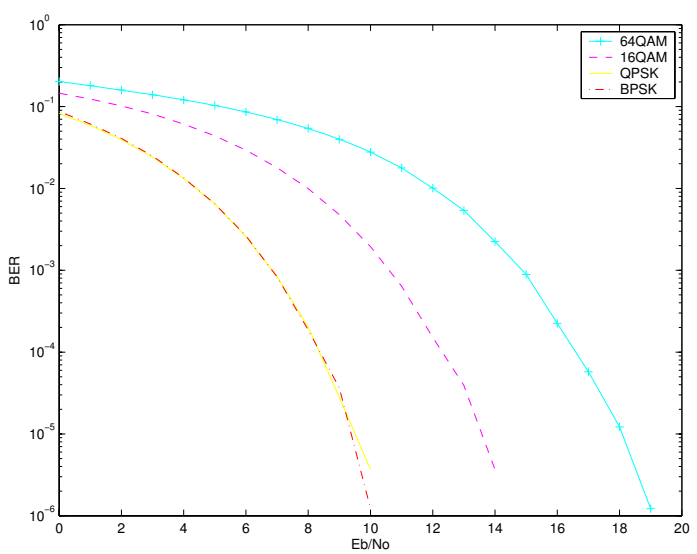

Fig. 8. Performance under Concurrent Tx/Rx in Gaussian Channel.

Performance under concurrent $\mathrm{Tx} / \mathrm{Rx}$ operation mode is also simulated in Gaussian channel. We assume that the local transmitter interference is $30 \mathrm{~dB}$ higher than the received signal and the remote transmitter interference is $0 \mathrm{~dB}$ relative to the received signal. We see in Fig 8 that performance in terms of bit-error-rate versus normalized signal-to-noise ratio is similar to that in normal operation mode. Note that under the same modulation scheme the actual data rate in concurrent $\mathrm{Tx} / \mathrm{Rx}$ mode will be only half of the data rate in the normal operation mode due to the use of outer code. However, the signal power in concurrent $\mathrm{Tx} / \mathrm{Rx}$ mode is also only half of the signal power in normal mode under the same normalized signal-to-noise ratio. Therefore, with the same signal power, the actual data rate in concurrent $\mathrm{Tx} / \mathrm{Rx}$ mode can be increased by using a higher rate modulation scheme.

\section{CONCLUSIONS}

This paper describes the development of an UWB PHY for mid-range high bit rate sensor networks for video surveillance in public safety and military applications. Future work will include development of medium access control (MAC) and networking layers QoS capability.

\section{ACKNOWLEDGMENT}

The authors acknowledge support of Motorola Inc. for funding the research described in this paper

\section{REFERENCES}

[1] Networking in Extreme Environments (NETEX), DARPA Program, http://www.darpa.mil/ato/programs/netex.htm.

[2] FCC Document, "Revision of Part 15 of the Commission's Rules Regarding Ultra-Wideband Transmission Systems", FCC 02-48, February 2002.

[3] Time Domain Corporation, "PulsON® Technology Overview," white paper, available on http://www.timedomain.com/products/ourtech/ whitepapers.html.

[4] R. Kohno et al, "DS-UWB Proposal Update", doc.: IEEE 802.15-04-0140-12-003a, March 2005, http://802wirelessworld.com/index.jsp.

[5] A. Batra et al, "Multi-band OFDM Physical Layer Proposal for IEEE 802.15 Task Group 3a", doc.: IEEE 802.15-04-0493-00-003a, September 2004, http://802wirelessworld.com/index.jsp.

[6] H-B. Li et al, "Merged Proposal of DS-UWB with Optional CS-UWB on UWB Band for IEEE 802.15.4a", doc.: IEEE 15-05-0127-01-004a, March 2005, http://802wirelessworld.com/index.jsp.

[7] A. Molisch et al, "UWB PHY Proposal for IEEE 802.15.4a Alt-PHY Project”, doc.: IEEE 802.15-050172-03-004a, 2005 , http://802wirelessworld.com/index.jsp.

[8] X. Huang and R. Jin, "Complementary-code-division multiplexing for future generation WLAN and mobile communication", 2004 International Symposium on Antennas and Propagation (ISAP2004), Sendai, Japan, August 17-21, 2004.

[9] X. Huang and Y..Li, "The multicode interleaved DSSS system for high speed wireless digital communications", in Proc. IEEE International Conf. Commun., vol. 10, pp. $2990-2994,2001$

[10] X. Huang, D. Lowe, R. Gandia, E. Dutkiewicz, “An Impulse Ultra-wideband System Capable of Concurrent Transmission and Reception, Part II: Design and Performance", International Conference on Communications, Circuits and Systems (ICCCAS2006), Gui Lin, China, June 25-28 2006. 